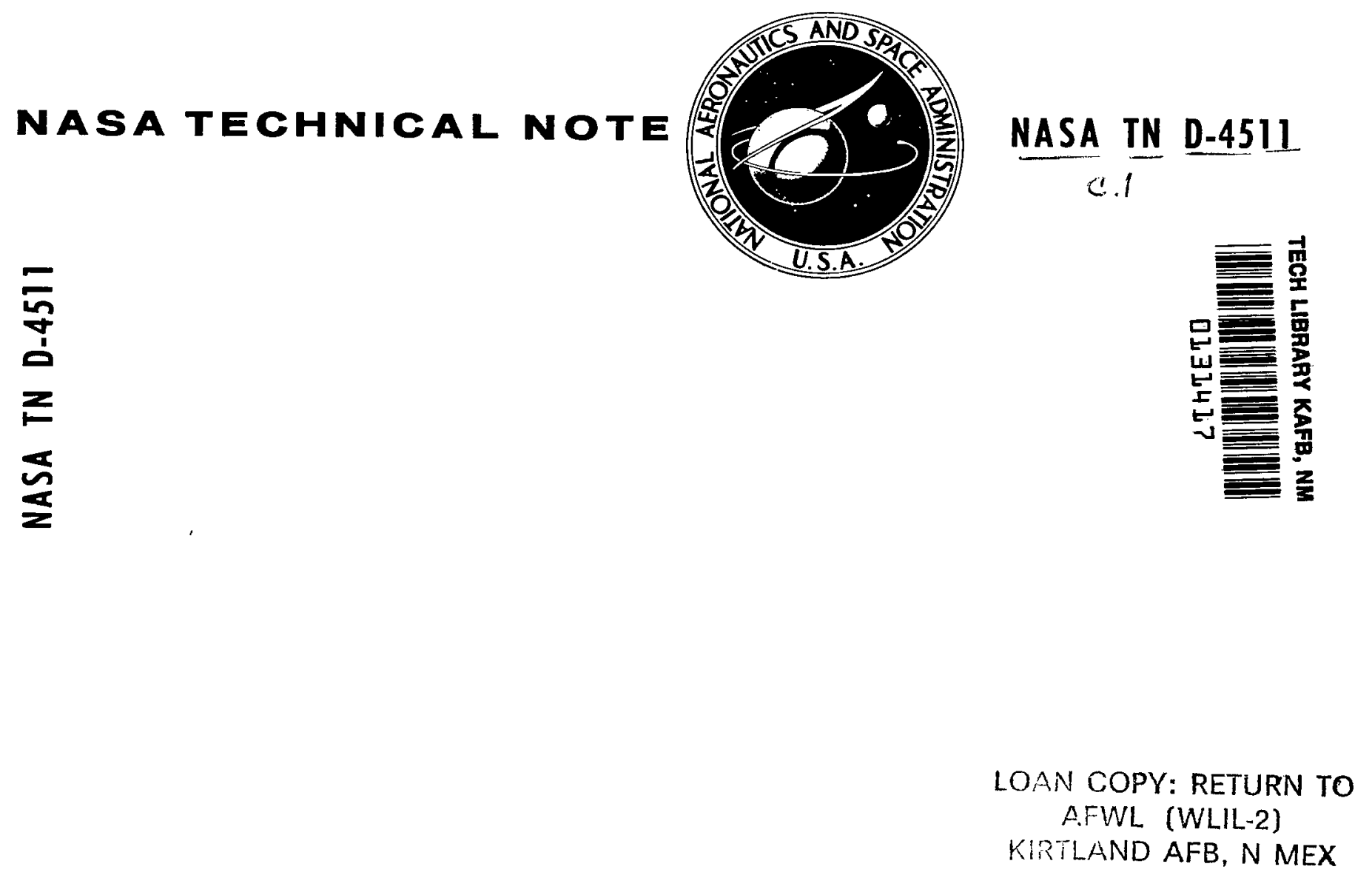

\title{
EXPLICIT UPPER BOUNDS \\ FOR ERROR PROPAGATION \\ IN THE n-BODY FIELD
}

by Albert Rosen

Goddard Space Flight Center Greenbelt, $M d$.

NATIONAL AERONAUTICS AND SPACE ADMINISTRATION - WASHINGTON, D. C. - AUGUST 1968 


\section{EXPLICIT UPPER BOUNDS FOR ERROR PROPAGATION}

IN THE n-BODY FIELD

By Albert Rosen

Goddard Space Flight Center

Gr eenbelt, Md.

\section{NATIONAL AERONAUTICS AND SPACE ADMINISTRATION}




\begin{abstract}
A simple means for bounding the propagated trajectory error induced by an impulsive initial error in the $n$-body field is developed. Formulas are derived for the maximum acceptable initial error, given the mission tolerances, and for the maximum time of negligible propagated error, given the initial impulses and mission tolerances. An example illustrates the use of the results within the solar system.
\end{abstract}




\title{
CONTENTS
}

\begin{abstract}
.................. ii
INTRODUCTION. . . . . . . . . . . . . . 1

AN INEQUALITY FROM THE EQUATIONS

OF MOTION ..................... 2

AN EXPLICIT UPPER BOUND FOR $|\overrightarrow{\mathrm{r}}|$ AND $|\vec{v}| \ldots 5$

References................... 13

Appendix A-Symbol List. . . . . . . . . . . . . . . 15
\end{abstract}




\title{
EXPLICIT UPPER BOUNDS FOR ERROR PROPAGATION IN THE n-BODY FIELD
}

\author{
by \\ Albert Rosen* \\ Goddard Space Flight Center
}

\section{INTRODUCTION}

One of the central problems of astronautical guidance is to estimate what effect a change in the position and velocity of a spacecraft at some initial time has on its position and velocity at some later time. Such a change may occur accidentally, as when there is an error in the value assigned to the initial state vector, or on purpose, as when a rocket motor induces an impulsive velocity change.

If the spacecraft has an essentially Kepler orbit, the propagation of the initial error can be analyzed precisely by matrix methods (References 1 and 2). If this error arises from a rocket motor, taking the cumulative velocity change as an impulse will cause the solution to be approximate. The closeness of approximation is discussed by Robbins (Reference 3). In circumstances where the Keplerian approximation is too crude, it is still possible to use matrix methods for the expression of error propagation, provided that the error is small enough to permit valid linearization of the relative force between the deviated and initial trajectory. Battin (Reference 4) and Baker (Reference 5) develop the proper forms for the matrices involved, and Dusek (Reference 6) gives a heuristic discussion of the validity of such linearization procedures.

Let the propagated error vectors be defined as the differences of the position and velocity from their values on the original orbit, at some time after the initial error is applied. Then, if it is desired to represent these vectors accurately, one of the above methods may be used.

It may be that certain errors are so small that they will produce no appreciable change on the trajectory, even after a considerable time. One way to verify this would be to include the effects in a numerical integration scheme and compare the resultant trajectory with a trajectory computed without considering the effects. Another way would be to use the linearized equations of motion in a matrix propagation. But it would be far simpler if there were some easy way to place an upper bound on the propagated effect of such a small error; then it should be enough to note that this upper bound is small (in view of the required precision).

*Student: St. Josephs College, Pennsylvania. 
We can develop a simple expression for such an upper bound by assuming that

1. The neglected error is approximated by the instantaneous variations $\vec{r}_{0}$ and $\vec{v}_{0}$ in position and velocity at time $\mathrm{t}=0$.

2. The motion of the spacecraft is adequately described by the n-body equations, for the time of interest:

3. Enough is known about the initial or "unperturbed" trajectory to permit placing lower bounds on the closest approach distances of this trajectory to each of the $n$ bodies, in the time interval under consideration.

As a measure of the effect of the error induced by the initial error $\vec{r}_{0}, \vec{v}_{0}$, in position and velocity, we define two vectors as follows: $\vec{r}(t)$ is the difference between positions at time $t$, in the perturbed and unperturbed orbits, respectively. $\vec{v}(t)$ is the same difference for velocities. At each time $t$, the vector $\vec{Q}_{i}$ will be the instantaneous vector to the $i^{\text {th }}$ body in the system, from the unperturbed trajectory.

\section{AN INEQUALITY FROM THE EQUATIONS OF MOTION}

Using the above definitions, Newton's Law of Gravitation leads at once to:

$$
\begin{gathered}
\ddot{\vec{r}}=\sum_{i=1}^{n} \mu_{i}\left[\frac{\vec{Q}_{i}-\vec{r}}{\left|\vec{Q}_{i}-\vec{r}\right|^{3}}-\frac{\vec{Q}_{i}}{\left|\vec{Q}_{i}\right|^{3}}\right], \\
\vec{r}(0)=\vec{r}_{0}, \\
\vec{v}(0)=\vec{v}_{0},
\end{gathered}
$$

where

$$
\begin{aligned}
& \mu_{i}=\text { gravitational constant of } i^{\text {th }} \text { body } \\
& n=\text { number of bodies in the system. }
\end{aligned}
$$

Eventually we will bound the magnitude of the solution of this highly nonlinear equation by the solution to a certain linear homogeneous scalar differential equation with constant coefficients, which can be derived from it.

Assume that $|\vec{r}(0)|<\left|\vec{Q}_{i}(0)\right|(i=1,2, \cdots, n)$. Then, for a short enough time, $|\vec{r}(t)|<\left|\vec{Q}_{i}(t)\right|$, because all these functions are continuous in time. 
We first show that $|\vec{r}(t)|<\left|\vec{Q}_{i}(t)\right|$ implies the inequality

$$
\left|\frac{\vec{Q}_{i}+\vec{r}}{\left|\vec{Q}_{i}+\vec{r}\right|^{3}}-\frac{\vec{Q}_{i}}{\left|\vec{Q}_{i}\right|^{3}}\right| \leq \frac{1}{\left(\left|\vec{Q}_{i}\right|-\mid \vec{r}\right)^{2}}-\frac{1}{\left|\vec{Q}_{i}\right|^{2}} .
$$

To verify this, expand the square of the quantity on the left, denoted by $U$, in powers of $\left|\vec{Q}_{i}\right| /|\vec{\rho}|$ and $|\vec{r}| /\left|\vec{Q}_{i}\right|$, where $\vec{\rho}=\vec{Q}_{\mathbf{i}}+\vec{r}$. The result is

$$
U^{2}=\frac{4}{\left|\vec{Q}_{i}\right|^{4}}\left[\frac{1}{4}-\frac{1}{4} \frac{\left|\vec{Q}_{i}\right|}{|\vec{\rho}|}+\frac{1}{4} \frac{\left|Q_{i}\right|^{4}}{|\vec{\rho}|^{4}}-\frac{1}{4} \frac{\left|Q_{i}\right|^{3}}{|\vec{\rho}|^{3}}\left(1-\frac{|\vec{r}|^{2}}{\left|\overrightarrow{Q_{i}}\right|^{2}}\right)\right] .
$$

The maximum value of $\mathrm{u}^{2}$ occurs when

$$
S(x)=\frac{1}{4}-\frac{1}{4} x+\frac{1}{4} x^{4}-\frac{1}{4} x^{3} a
$$

maximizes, $x=\left|\vec{Q}_{i}\right| /|\vec{\rho}|$, a $a-|\vec{r}|^{2} /\left|\vec{Q}_{i}\right|^{2}$ and the magnitudes $\left|\vec{Q}_{i}\right|$ and $|\vec{r}|$ are fixed. Since $|\vec{r}|<\left|\vec{Q}_{\mathbf{i}}\right|$, the inequality 4 restricts $\alpha$ and $\mathrm{x}$ :

$$
0<\alpha \leq 1 \text { and } \frac{1}{1+\sqrt{(1-a)}} \leq \mathrm{x} \leq \frac{1}{1-\sqrt{(1-a)}}
$$

with the extreme values of $x$ in inequality 4 corresponding to $\vec{r}$ parallel and antiparallel to $\vec{Q}_{i}$.

But the derivative of $S(x)$ is

$$
S^{\prime}(x)=x^{3}-\frac{3}{4} a x^{2}-\frac{1}{4}
$$

If we equate $S^{\prime}(x)$ to zero and solve for $x$, the discriminant is $\left(a^{3}+4\right) / 256$, which must be positive, according to inequality 4 . Hence $S^{\prime}(x)=0$ has one real root. Evaluating $S^{\prime}(x)$ for the extreme values of $\mathrm{x}$ in inequality 4 gives

$$
S^{\prime}\left(\frac{1}{1+\sqrt{1-a}}\right)=-\frac{\sqrt{1-a}\left(1+\frac{a}{2}\right)}{(1+\sqrt{1-a})^{3}}, \text { which is negative, }
$$

and

$$
S^{\prime}\left(\frac{1}{1-\sqrt{1-a}}\right)=\frac{\sqrt{1-a}\left(1+\frac{a}{2}\right)}{(1-\sqrt{1-a})^{3}}, \text { which is positive }
$$

so that the single real root for $S^{\prime}(x)=0$ lies inside the interval of $x$ given by inequality 4 . 
This means that $S(x)$ takes on local maximum values for $x=1 /(1+\sqrt{1-\alpha})$ and $x=1 /(1-\sqrt{1-a})$ because, where $S^{\prime}(x)=0$, we have an absolute minimum for $S(x)$. Consider that $S(x) \rightarrow \infty$ as $x \rightarrow \pm \infty$.

Hence, either for $\vec{r}$ parallel to $\vec{Q}_{i}$ (giving $U=U_{p}$ ) or for $\vec{r}$ antiparallel to $\vec{Q}_{i}$ (giving $U=U_{a}$ ), $U$ takes on its maximum value. Evaluating $U$ for these two orientations of $\vec{r}$ and $\vec{Q}_{i}$ gives

$$
\begin{gathered}
U_{a}=\frac{1}{\left(\left|\vec{Q}_{i}\right|-|\vec{r}|\right)^{2}}-\frac{1}{\left|\vec{Q}_{i}\right|^{2}} \equiv \frac{1}{\left|\vec{Q}_{i}\right|^{2}}\left[2 \frac{|\vec{r}|}{\left|\vec{Q}_{i}\right|}+3 \frac{|\vec{r}|^{2}}{\left|\vec{Q}_{i}\right|^{2}}+\cdots(n+1) \frac{|\vec{r}|^{n}}{\left|\vec{Q}_{i}\right|^{n}}+\cdots\right], \\
U_{p}=-\frac{1}{\left(\left|\vec{Q}_{i}\right|-|\vec{r}|\right)^{2}}+\frac{1}{\left|\vec{Q}_{i}\right|^{2}} \equiv \frac{1}{\left|Q_{i}\right|^{2}}\left[2 \frac{|\vec{r}|}{\left|\vec{Q}_{i}\right|}-3 \frac{|\vec{r}|^{2}}{\left|\overrightarrow{Q_{i}}\right|^{2}}+\cdots(-1)^{(n+1)}(n+1) \frac{|\vec{r}|^{n}}{\left|\vec{Q}_{i}\right|^{n}}+\cdots\right],
\end{gathered}
$$

so that $U_{a} \geq U_{p}$, and $U_{a}$ is an upper bound for $U$ for all orientations of $\vec{r}$ and $\vec{Q}_{i}$. This completes the proof of inequality 2 .

Returning to Equation 1, the triangle inequality can be combined with inequality 2 to give the result

$$
|\ddot{\vec{r}}| \leq \sum_{i=1}^{n} \mu_{i}\left\{\frac{1}{\left(\left|\vec{Q}_{i}\right|-|\vec{r}|\right)^{2}}-\frac{1}{\left|\vec{Q}_{i}\right|^{2}}\right\},
$$

provided that $|\overrightarrow{\mathbf{r}}|<\left|\vec{Q}_{\mathbf{i}}\right|$. This is identical with the inequality

$$
|\ddot{\vec{r}}| \leq|\vec{r}| f^{2}\left(|\vec{r}|,\left|\vec{Q}_{i}\right|\right)
$$

where $f$ is a function of the $n+1$ variables $x, y_{1}, y_{2}, \cdots, y_{n}$ given by

$$
f\left(x, y_{i}\right)=\left[\sum_{i=1}^{n} \frac{2 \mu_{i}}{y_{i}^{3}} \cdot \frac{\left(1-\frac{1}{2} \frac{x}{y_{i}}\right)}{\left(1-\frac{x}{y_{i}}\right)^{2}}\right]^{1 / 2} .
$$

It is assumed in Equation 11 that $0 \leq x<y_{i}(i=1,2, \cdots, n)$. Clearly $f$ and $f^{2}$ are monotonically increasing functions of $x$. Also, $f \partial f / \partial x$ is a positive monotonically increasing function of $x$, because it is given by

$$
f\left(x, y_{i}\right) \frac{\partial f}{\partial x}\left(x, y_{i}\right)=\sum_{i=1}^{n} \frac{\mu_{i}}{y_{i}{ }^{4}} \frac{\left(3-\frac{x}{y_{i}}\right)}{\left(1-\frac{x}{y_{i}}\right)^{3}} .
$$


Both $f\left(x, y_{i}\right)$ and $f\left(x, y_{i}\right) \partial f\left(x, y_{i}\right) / \partial x$ are monotonically decreasing in all the $y_{i}{ }^{\prime} s$, so long as $x$ remains positive and less than each of these variables.

For sufficiently small $t$, inequality 10 is valid and can be combined with

$$
|\vec{r}| \leq \int_{0}^{t} \int_{0}^{t}|\ddot{\vec{r}}(s)| d s^{2}+\left|\vec{r}_{0}\right|+\left|\vec{v}_{0}\right| \cdot t
$$

which is true for all $t$, to give:

$$
|\vec{r}| \leq \int_{0}^{t} \int_{0}^{t}|\vec{r}(s)| f^{2}\left(|\vec{r}|,\left|\vec{Q}_{i}\right|\right) d s^{2}+\left|\vec{r}_{0}\right|+\left|\vec{v}_{0}\right| \cdot t .
$$

This implicit integral equality enables us to get an explicit upper bound on $|\vec{r}|$ and $|\vec{v}|$.

\section{AN EXPLICIT UPPER BOUND FOR $|\vec{r}|$ AND $|\vec{v}|$}

Consider the differential equations

$$
\begin{aligned}
\ddot{w} & =w^{2}\left(x, c_{i}\right) \\
w(0) & =\left|\vec{r}_{0}\right| \\
\dot{w}(0) & =\left|\vec{v}_{0}\right| .
\end{aligned}
$$

For the present, $x$ is a parameter which has the range $0 \leq x<C_{m i n}$, where $C_{m i n}$ is the smallest of the $C_{i}$ 's. We assume that the $C_{i}$ 's are chosen on some interval $0 \leq t \leq T_{1}$ in such a way that

$$
C_{i} \leq\left|\vec{Q}_{i}(t)\right| ; \quad 0 \leq t \leq T_{1}
$$

and we restrict $\vec{r}_{0}$ to satisfy the relation

$$
0 \leq\left|\vec{r}_{0}\right|<C_{\text {min }} .
$$

This last restriction causes no practical difficulties, because the $C_{i}$ represent lower bounds on the closest approach of the unperturbed trajectory to the $i^{\text {th }}$ body, and no initial error in position will in practice be as large as any of these distances.

The solution of Equation 15 on $0 \leq t \leq T_{1}$ is given by

$$
w=\left|\vec{r}_{0}\right| \cosh f\left(x, C_{i}\right) t+\frac{\left|\vec{v}_{0}\right| \sinh f\left(x, C_{i}\right) t}{f\left(x, C_{i}\right)}
$$


which satisfies the integral equation

$$
\begin{gathered}
w(x, t)=\int_{0}^{t} \int_{0}^{t} w(s) f^{2}\left(x, C_{i}\right) d s^{2}+\left|\vec{r}_{0}\right|+\left|\vec{v}_{0}\right| t \\
0 \leq t \leq T_{1} .
\end{gathered}
$$

Comparing Equation 19 and inequality 14 leads us to suppose that $w(x, t)$ may provide an upper bound for $|\vec{r}(t)|$, provided that $x$ can be chosen as an upper bound on $|\vec{r}(t)|$, during the time under consideration. If this were so, we might try to choose $\mathrm{x}$ to satisfy

$$
w(x, T) \leq x ; \quad T \leq T_{1},
$$

which would be consistent, at least, with the supposition that $w(x, t)$ provides an upper bound on $|\vec{r}|$ on $0 \leq t \leq T, T \leq T_{1}$, and that $x$ provides an upper bound on $|\vec{r}|$ on that interval.

These heuristic considerations lead to the following theorem.

Theorem I. Let $w(x, t)$ be defined as in Equation 18, let the $C_{i}$ 's be chosen to satisfy Equation 16, and let $\left|\vec{r}_{0}\right|$ be restricted as in inequality 17 . Then if $x$ satisfies inequality $20: w(x, t)$ provides an upper bound on $|\vec{r}|$ on $0 \leq t \leq T$, and $\partial w(x, t) / \partial t$ provides an upper bound on $|\vec{v}|$ on that interval.

We show that $w(x, t)$ provides an upper bound on $|\vec{r}|$ on the half-open interval $0 \leq t<T$. The continuity of $|\vec{r}|$ and $w(x, t)$ in time then enables the extension of this result to the endpoint of that interval.

Assume the contrary, that there is a time in $0 \leq t<T$ such that $w(x, t)<|\vec{r}(t)|$. Then, by the continuity of $|\vec{r}|$ and $w$ in time, there is an open subinterval of $0 \leq t<T$ where $w(x, t)<|\vec{r}(t)|$, and there is a first such subinterval. Letting $t_{1}$ denote the beginning of the subinterval, $\gamma(t) \equiv|\vec{r}(t)|$ $-w(x, t)$, and letting $t_{2}$ be some time $>t_{1}$, sufficiently close to $t_{1}$, gives the inequalities:

$$
\begin{array}{ll}
\text { (a) } \gamma(\mathrm{t}) \leq 0 & 0 \leq \mathrm{t} \leq \mathrm{t}_{1} \\
\text { (b) } \gamma(\mathrm{t})=0 & \mathrm{t}=\mathrm{t}_{1} \\
\text { (c) } \gamma(\mathrm{t})>0 & \mathrm{t}_{1}<\mathrm{t} \leq \mathrm{t}_{2} \\
\text { (d) }|\overrightarrow{\mathrm{r}}| \leq \mathrm{x} & 0 \leq \mathrm{t} \leq \mathrm{t}_{2} \\
\text { (e) } \gamma\left(\mathrm{t}_{2}\right) \geq \gamma(\mathrm{t}) & \mathrm{t}_{1} \leq \mathrm{t} \leq \mathrm{t}_{2} \\
\text { (f) } \mathrm{t}_{1}<\mathrm{t}_{2}<\mathrm{T} . &
\end{array}
$$

To see that inequality $21 \mathrm{~d}$ is true, note that inequality $21 \mathrm{~b}$ implies that $\left|\vec{r}\left(t_{1}\right)\right|<x$, because $w\left(x, t_{1}\right)<w(x, T) \leq x$. Continuity then demands that $|\vec{r}(t)|$ remain less than or equal to $x$ for a short enough time after $t_{1}-i$.e., for a $t_{2}$ close enough to $t_{1}$. 
Inequality 21e follows from the fact that

$$
\gamma(\mathrm{t})=\int_{\mathrm{t}_{1}}^{\mathrm{t}} \dot{\gamma}(\mathrm{s}) \mathrm{ds}, \quad \mathrm{t}_{1} \leq \mathrm{t} \leq \mathrm{t}_{2} .
$$

since $\gamma\left(t_{1}\right)=0$. But since this is positive for every $t$ in $t_{1}<t \leq t_{2}$, then $\dot{\gamma}(s)$ must be positive in that interval, so $\gamma(t)$ is monotonically increasing in $t$ in that interval.

Since inequality $21 d$ is true, and $x \leq\left|\vec{Q}_{i}(t)\right|$ on $0 \leq t \leq T$, we conclude that inequality 14 must hold in $0 \leq t<t_{2}$. Subtracting Equation 19 from inequality 14 then gives, letting $t=t_{2}$,

$$
0<\gamma\left(t_{2}\right) \leq \int_{t_{1}}^{t_{2}} \int_{t_{1}}^{t_{2}}\left[|\vec{r}(s)| f^{2}\left(|\vec{r}(s)|,\left|\vec{Q}_{i}(s)\right|\right)-w(x, s) f^{2}\left(x, c_{i}\right)\right] d s^{2},
$$

since the contribution to the integral from $0 \leq t \leq t_{1}$ is not positive. But by inequality $21 d$, and the fact that $c_{i} \leq\left|\vec{Q}_{i}\right|(i=1,2, \ldots, n)$, we can reduce this to

$$
0<\gamma\left(t_{2}\right) \leq f^{2}\left(x, C_{i}\right) \int_{t_{1}}^{t} \int_{t_{1}}^{t_{2}}[|\vec{r}(s)|-w(x, s)] d s^{2} .
$$

The mean-value theorem then gives, with $t_{1} \leq t_{3} \leq t_{2}$ :

$$
0<\gamma\left(t_{2}\right) \leq f^{2}\left(x, C_{i}\right) \cdot \gamma\left(t_{3}\right)\left(t_{2}-t_{1}\right)^{2}
$$

But by inequality $21 \mathrm{e}$, this implies that

$$
0<\gamma\left(t_{2}\right) \leq f^{2}\left(x, C_{i}\right) \gamma\left(t_{2}\right)\left(t_{2}-t_{1}\right)^{2}
$$

This is a contradiction, as may be seen by dividing through by $\gamma\left(t_{2}\right)$ and letting $\left(t_{2}-t_{1}\right)$ approach zero. Therefore $w(t) \geq|\vec{r}|$ on $0 \leq t<T$ and, by continuity, on $0 \leq t \leq T$.

To see that $\partial \mathrm{w} / \partial \mathrm{t} \geq|\overrightarrow{\mathrm{v}}|$ on $0 \leq \mathrm{t}<\mathrm{T}$, we use this result, noting that $\mathrm{f}^{2}\left(|\overrightarrow{\mathrm{r}}|,\left|\overrightarrow{\mathrm{Q}}_{\mathrm{i}}\right|\right) \leq \mathrm{f}^{2}\left(\mathrm{x}, \mathrm{C}_{\mathrm{i}}\right)$ on $0 \leq t \leq T$, and also that inequality 16 is true on $0 \leq t \leq T$. But, always:

$$
|\vec{v}| \leq \int_{0}^{t}|\ddot{\vec{r}}(s)| d s+\left|\vec{r}_{0}\right|
$$


Taking the first partial derivative of Equation 19 with respect to time, and combining this with inequalities 26 and 10 gives

$$
|\vec{v}|-\frac{\partial w(x, t)}{\partial t} \leq \int_{0}^{t}\left[|\vec{r}| f^{2}\left(|\vec{r}|,\left|\vec{Q}_{i}\right|\right)-w f^{2}\left(x, C_{i}\right)\right] d s
$$

which by the above remarks is reducible to

$$
|\vec{v}|-\frac{\partial w(x, t)}{\partial t} \leq f^{2}\left(x, C_{i}\right) \int_{0}^{t}[|\vec{r}(s)|-w(x, s)] d s .
$$

Since the integrand has been shown to be non-positive for all $t$ in $0 \leq t \leq T$, the left-hand side of inequality 28 must also be non-positive in that interval. This concludes the proof of theorem I.

The question now is: for what range of values of $T,\left|\vec{r}_{0}\right|$, and $\left|\vec{v}_{0}\right|$ does there exist a range of $x$ values that satisfy inequality 20 , and what is this range of $x$ values? Can these $x$-values be conveniently found?

Consider the following expressions for $w(x, T)$ and $\partial w(x, T) / \partial x$ :

$$
\begin{gathered}
w(x, T)=\left|\vec{r}_{0}\right| \cosh \mathrm{fT}+\vec{v}_{0} \frac{\sinh f T}{f} \\
=\left|\vec{r}_{0}\right|+\left|\vec{v}_{0}\right| T+0\left(T^{2}\right) \\
\frac{\partial w(x, T)}{\partial x}=\left|\vec{r}_{0}\right| T^{2}\left(f f^{\prime}\right)\left(\frac{\sinh f T}{f T}\right)+\left|\vec{v}_{0}\right| T^{3}\left(f f^{\prime}\right) \frac{1}{(f T)^{2}}\left(\cosh f T-\frac{\sinh f T}{f T}\right) \\
=\left|\vec{r}_{0}\right| T^{2}\left(f f^{\prime}\right)\left\{1+\frac{(f T)^{2}}{3 !}+\frac{(f T)^{4}}{5 !}+\cdots\right\} \\
+\left|\vec{v}_{0}\right| T^{3}\left(f f^{\prime}\right)\left[\left(\frac{1}{2 !}-\frac{1}{3 !}\right)+(f T)^{2}\left(\frac{1}{4 !}-\frac{1}{5 !}\right)+(f T)^{4}\left(\frac{1}{6 !}-\frac{1}{7 !}\right)+\cdots\right] .
\end{gathered}
$$

In Equations 29 and $30, f=f\left(x, C_{i}\right)$ and $f^{\prime}=\partial f\left(x, C_{i}\right) / \partial x$.

Both series in Equation 30 are monotonically increasing in $f T$, and both $f$ and $f f^{\prime}$ are monotonically increasing in $\mathrm{x}$; therefore $\partial \mathrm{w}(\mathrm{x}, \mathrm{T}) / \partial \mathrm{x}$ is monotonically increasing in $\mathrm{x}$ and $\mathrm{T}$.

When $x=\left|\vec{r}_{0}\right|, w(x, T)-x$ is positive for $T>0$, and $w(x, T)-x$ is also positive for $x \rightarrow C_{m i n}$, because this makes $f\left(x, C_{i}\right)$ infinite. The fact that $\partial w(x, T) / \partial x$ is positive and monotonically increasing in $x$ implies that the function $w(x, T)-x$ is always curving upward in $x$, so that it equals zero at 
none, one, or two points. If there are any roots, then $w(x, T)-x$ has a minimum which is also an extremum on $\left|\vec{r}_{0}\right| \leq x<C_{m i n}$. Clearly then, it is possible to find $x$ such that $w(x, T) \leq x$ is satisfied if and only if there are real roots to

$$
w(x, T)-x=0 ; \quad\left|\vec{r}_{0}\right| \leq x<C_{\text {min }},
$$

and any value of $\mathrm{x}$ between or including these roots will satisfy $w(x, T) \leq x$.

For small T, Equation 31 has two roots, since the series in Equation 29 can be made arbitrarily close to $\left|\vec{r}_{0}\right|$ by choosing a small enough $T$, regardless of where $x$ is in $\left|\vec{r}_{0}\right| \leq x<C_{m i n}$. For a large $T$, the value of $\partial w\left(x, C_{i}\right) / \partial x$ at $x=\left|\vec{r}_{0}\right|$ becomes large, and since it is always increasing in $x$ on $\left|\vec{r}_{0}\right| \leq x<C_{\min }$, there can be no intersection points of $w(x, T)$ with $x$; and no roots to $w(x, T)-x=0$. There is a critical value of $\mathrm{T}$, therefore, which permits one and only one solution to Equation 31 , and this is the largest $T$ for which inequality 20 can be satisfied. At this critical value of $T$, the root of Equation 31 defines the extremum of $w(x, T)-x$, so the condition

$$
\frac{\partial}{\partial x}[w(x, T)-x]=0, \quad\left|\vec{r}_{0}\right| \leq x<C_{\text {min }}
$$

must also be satisfied. Solving Equations 31 and 32 simultaneously for $\mathrm{T}$ gives the desired critical value, provided that we know $\left|\vec{v}_{0}\right|,\left|\vec{r}_{0}\right|$, and all the $C_{i}$ 's.

A simpler method for finding the critical $\mathrm{T}$ can be derived, however. The function $w(x, T)$ has the form $w=w(f T, f)$. The total derivative of Equation 31 with respect to $x$ is therefore given by

$$
\frac{\partial w(x, T)}{\partial(\tilde{f} T)}\left[\frac{\partial f}{\partial x} \cdot T+\frac{d T}{d x} \cdot f\right]+\frac{\partial w(x, T)}{\partial f} \cdot \frac{\partial f}{\partial x}=1
$$

where we use, the fact that Equation 31 determines $\mathrm{T}$ as a function of $\mathbf{x}$. On the other hand, Equation 32 is identical with

$$
\frac{\partial w}{\partial(f T)}\left[\frac{\partial f}{\partial x} \cdot T\right]+\frac{\partial w}{\partial f} \frac{\partial f}{\partial x}=1
$$

Since there is a unique solution to Equations 31 and 32 on $\left|\vec{r}_{0}\right| \leq x<C_{m i n}$, and since $f \cdot(\partial w / \partial(f T))$ is readily verified to be nonzero, we conclude that Equation 31 combined with Equation 32 is the same as Equation 31 combined with the condition that $\mathrm{dT} / \mathrm{dx}=0$. This condition determines the maximum value of $T(x)$ on $\left|\vec{r}_{0}\right| \leq x<C_{m_{i}}$, because $T$ must vanish at both endpoints, in order that Equation 31 shall have roots. Solving Equation 31 for $T$ explicitly leads to the following theorem. 
Theorem II. Let $\tau(\mathrm{x})$ be defined by

$$
\tau(x)=\frac{1}{f\left(x, C_{i}\right)} \ln \left\{\frac{x+\sqrt{x^{2}-\left|\vec{r}_{0}\right|^{2}+\left[\frac{\left|\vec{v}_{0}\right|}{f\left(x, C_{i}\right)}\right]^{2}}}{\left|\vec{r}_{0}\right|+\frac{\left|\vec{v}_{0}\right|}{f\left(x, C_{i}\right)}}\right\} .
$$

Then, for all $\mathrm{T}$ satisfying the inequality

$$
\mathrm{T} \leq \max (\tau(\mathrm{x})), \quad\left|\overrightarrow{\mathrm{r}}_{0}\right| \underline{\underline{x}} \mathbf{x}<\mathrm{C}_{\mathrm{min}},
$$

there are values of $x$ satisfying $w(x, T) \leq x$; all such values lie in the interval $x_{1} \leq x \leq x_{2}$, where $x_{1}$ and $x_{2}$ are the two roots of the transcendental equation:

$$
\left|\vec{r}_{0}\right| \cosh f\left(x, C_{i}\right) T+\frac{\left|\vec{v}_{0}\right|}{f\left(x, C_{i}\right)} \sinh f\left(x, C_{i}\right) T=x,
$$

on $\left|\vec{r}_{0}\right| \leq x<C_{\text {min }}$.

Suppose that the impulse $\vec{v}_{0}$ and position error $\vec{r}_{0}$ were assigned and it was required to know for how.long a time the propagated effect of these vectors would remain less than $\mathrm{x}$ in position magnitude $\left(\left|\vec{r}_{0}\right| \leq x<C_{m i n}\right)$. The answer to this is: set $T=\tau(x)$. This is legitimate, because, if $\mathrm{T}=\tau(\mathrm{x})$, then Equation 37 is satisfied, so $w(x, T)=x$. Hence inequality 20 is satisfied, and $w(x, t)$ bounds the error in position on $0 \leq t \leq T$, and $x$ bounds the error in position on $0 \leq t \leq T$ also, as needed, since $x \geq w(x, t)$ on $0 \leq t \leq T$.

Another theorem may be presented here:

Theorem III. If it is required that the propagated position error be less than or equal to $\mathrm{x}$, where $\left|\vec{r}_{0}\right| \leq x<C_{m i n}$, then this is assured by taking the time of propagation equal to $\tau(x)$, where $\tau(x)$ is defined in Equation 35.

Suppose, next, that it is desired to know how large an initial error, $\vec{r}_{0}$ and $\vec{v}_{0}$ is permissible, in order that the propagated effects of these errors over a pre-assigned time $T$ should be less than $\mathbf{x}\left(\left|\vec{r}_{0}\right| \leq x<C_{\min }\right)$.

To answer this, note that for any $\mathrm{x}$ satisfying $0 \leq \mathrm{x}<\mathrm{C}_{\mathrm{m} i \mathrm{n}}$, it is possible to choose the coefficients of $\cosh f\left(x, C_{i}\right) T$ and $\left[\sinh f\left(x, C_{i}\right) T\right] / f\left(x, C_{i}\right)$ so that

$$
\left|\vec{r}_{0}\right|\left(\cosh f\left(x, C_{i}\right) \cdot T\right)+\left|\vec{v}_{0}\right|\left(\frac{\sinh f\left(x, C_{i}\right) T}{f\left(x, C_{i}\right)}\right) \leq x
$$

is satisfied, thereby guaranteeing that $x$ is indeed an upper bound on $|\vec{r}|$ after time T. In particular, if $\left|\vec{r}_{0}\right|=0$, then the propagated error is less than $\times$ after time $T$, provided that $\left|\vec{v}_{0}\right|$ satisfies the 
inequality

$$
\left|\vec{v}_{0}\right| \leq \frac{\operatorname{xf}\left(x, C_{i}\right)}{\sinh \left(f\left(x, C_{i}\right) T\right)}
$$

Note that although $\tau(\mathrm{x})$ in Equation 35 may become formally greater than $\mathrm{T}_{1}$, the time for which the $C_{i}{ }^{~}$ 's were determined, the fact has no physical significance. If this happens, we can only be assured that $x$ exists to satisfy inequality 20 on $0 \leq t \leq T_{1}$. If it is desired to extend the interval past $T_{1}$, then it is necessary to find $C_{i}$ 's for this extended interval to make all the results valid.

It is also worth noting that looser tolerances in mission requirements (larger $\mathrm{x}$ ) in Theorem III do not necessarily produce larger times of negligible effect, because $\tau(x)$ does have a maximum. If $x$ is chosen to be larger than the value which maximizes $\tau(x)$, then a looser tolerance will decrease $\tau(x)$, giving pessimistic results. In such a case, the solution is to take the time as maximum $\tau(\mathbf{x})$, since the error $x$ corresponding to this maximum is certainly less than an error corresponding to the times less than the maximum, brought on by large $x$.

Finally, if $x / C_{m i n}$ is small, then, to a good approximation, $f\left(x, C_{i}\right)=f\left(0, C_{i}\right)$, i.e.,

$$
\mathrm{f}\left(\mathrm{x}, \mathrm{C}_{\mathrm{i}}\right) \approx \sqrt{\sum_{\mathrm{i}=1}^{\mathrm{n}} \frac{2 \mu_{\mathrm{i}}}{\mathrm{C}_{\mathrm{i}}{ }^{3}}}, \quad \text { if } \quad \mathrm{x} / \mathrm{C}_{\mathrm{min}} \ll 1
$$

\section{Example}

A spacecraft enroute to the outer planets passes through the asteroid belt and may be subjected to an impulsive velocity change there as large as $50 \mathrm{~km} /$ day $=5.8 \times 10^{-4} \mathrm{~km} / \mathrm{sec}$. This figure may have been arrived at by placing lower bounds on the closest approach of the spacecraft to the asteroids (as determined by their physical radius), lower bounds on the relative velocity of rocket and asteroid, and upper bounds on the masses of asteroids, and by assuming Keplerian interactions for the short encounter time with the asteroid. We wish to examine the orbit for some part of its path during which the closest approach distances to the sun and other large bodies in the solar system is given by Table 1.

The other bodies in the solar system may be considered negligible in influencing the propagation of the impulse. For how long does the propagated error in position remain less than 0.008 AU?

Solution: Use Theorem III. First calculate $f\left(x, C_{i}\right)$. Since $0.008 / c_{i} \ll 1$ by Table 1 for all the bodies, we use the approximation:

$$
\mathrm{f}\left(\mathrm{x}, \mathrm{C}_{\mathrm{i}}\right) \approx \sqrt{\sum \frac{2 \mu_{\mathrm{i}}}{\mathrm{C}_{\mathrm{i}}^{3}}} \equiv\left\{\frac{2 \mu_{\mathrm{s}}}{\mathrm{C}_{\mathrm{s}}^{3}}\left[1+\sum_{\mathrm{i}=2}^{9}\left(\frac{\mu_{\mathrm{i}}}{\mu_{\mathrm{s}}}\right)\left(\frac{\mathrm{C}_{\mathrm{s}}}{\mathrm{C}_{\mathrm{i}}}\right)^{3}\right]\right\}^{1 / 2},
$$

where $\mu_{s}=$ Sun's gravitational parameter. 
Table 1

Closest Approach of Spacecraft to Bodies in Solar System

\begin{tabular}{|l|c|}
\hline Body & Closest Approach, AU* \\
\hline Sun & 1.50 \\
Mercury & 1.00 \\
Venus & 1.60 \\
Earth & 1.00 \\
Mars & 0.80 \\
Jupiter & 3.50 \\
Saturn & 8.00 \\
Uranus & 9.00 \\
Neptune & 14.00 \\
\hline
\end{tabular}

Now the largest value of $\mu_{i} / \mu_{s}$ is that for Jupiter, which is $0.9 \times 10^{-3}$. Also, by Table 1 , the largest value of $\left(\mathrm{C}_{\mathrm{s}} / \mathrm{C}_{\mathrm{i}}\right)^{3}$ is that for Mars, which is 6.6. Hence, to a percent accuracy,

$$
f\left(x, C_{i}\right)=\sqrt{\frac{2 \mu_{s}}{C_{s}^{3}}},
$$

where $\mu_{s}$ is in $\mathrm{AU}^{3}$ days ${ }^{-2}, \mathrm{C}_{\mathrm{s}}$ is in $\mathrm{AU}$. That is:

$$
\begin{aligned}
f\left(x, C_{i}\right) & =\sqrt{\frac{5.92 \times 10^{-4}}{(1.50)^{3}}} \\
& =\frac{1}{44.5} \text { days }^{-1} .
\end{aligned}
$$

Therefore we are sure of a propagated error less than 0.008 (or $1.20 \times 10^{5} \mathrm{~km}$ ) for a length of time given by

$$
\begin{aligned}
T & =\tau\left(1.20 \times 10^{6}\right) \\
& =44.5 \ln \left\{\frac{1.20 \times 10^{6}+\sqrt{\left(1.20 \times 10^{6}\right)^{2}+(50.0 \times 44.5)^{2}}}{50.0 \times 44.5}\right\} \\
& =44.5 \ln \left\{\frac{1.20 \times 10^{6}}{25.0 \times 44.5}\right\} \\
& =44.5 \ln (1080)=44.5 \times 6.98=311 \text { days. }
\end{aligned}
$$

As another example, suppose we wish to know for how large a velocity impulse the propagated effect in position would be less than 0.008 AU over a period of 100 days, assuming that Table 1 is valid.

Solution: Again, $f\left(x, c_{i}\right)=1 / 44.5$ and we can use inequality 39 to obtain the upper bound on $\left|\vec{v}_{0}\right|$ :

$$
\begin{aligned}
\left|\vec{v}_{0}\right| & \leq \frac{1.2 \times 10^{6}}{44.5 \sinh \left(\frac{100}{44.5}\right)} \\
& =\frac{1.2 \times 10^{6}}{44.5 \sinh (2.24)} \\
& =\frac{1.2 \times 10^{6}}{44.5 \cdot 4.64}
\end{aligned}
$$




$$
\begin{aligned}
& =\frac{1.20 \times 10^{6}}{206} \\
& =5811 \frac{\mathrm{km}}{\mathrm{day}} \\
& =067 \mathrm{~km} / \mathrm{sec} .
\end{aligned}
$$

So impulses as large as $0.06 \mathrm{~km} / \mathrm{sec}$ could be tolerated without violating mission requirements.

\section{ACKNOWLEDGMENT}

The author would like to thank Dr. Peter Musen and Dr. Peter Argentiero of Goddard Space Flight Center for their encouragement and suggestions.

Goddard Space Flight Center

National Aeronautics and Space Administration

Greenbelt, Maryland, December 28, 1967

188-63-01-01-51

\section{REFERENCES}

1. Goodyear, W. H., and Guseman, L. F., "A Modification of Herrick's Solution of the Two Body Problem for all Cases," IBM/RTCC Rept. 12-008, 1964.

2. Danby, J. M. A., "Matrizant of Keplerian Motion," AIAA Journal 3(4):769-770, April 1965.

3. Robbins, H. M., "An Analytical Study of the Impulsive Approximation," AIAA Journal 4(8):14171423, August 1966.

4. Battin, R. H., "Astronautical Guidance," Chap. VI, New York: McGraw-Hill, 1964, pp. 203-211.

5. Baker, R. M. L., "Astrodynamics-Applications and Advanced Topics," Chap. 1, New York: Academic Press, 1967, pp. 82-103.

6. Dusek, H. M., "Applicability of Linear Perturbation Theory in Midcourse Guidance," ARS Journal 32(8):1287-1289, August 1962. 


\section{Appendix A}

\section{Symbol List}

$\vec{r}_{0}, \vec{v}_{0}$ : Initial errors in position and velocity.

$\vec{r}(t), \vec{v}(t)$ : Instantaneous errors in position and velocity.

$\vec{Q}_{i}(t)$ : Vector from unperturbed orbit to $i^{\text {th }}$ body.

.$\mu_{i}$ : Gravitational parameter of $i^{\text {th }}$ body.

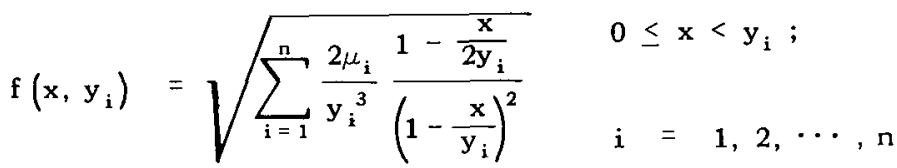

$C_{i}$ : Upper bound on $\left|\vec{Q}_{i}(t)\right|$ on $0 \leq t \leq T_{1}$.

$C_{m i n}$ : Smallest of the $C_{i}$ 's.

$w(x, t) \equiv\left|\vec{r}_{0}\right| \cosh f\left(x, C_{i}\right) t+\frac{v_{0}}{f\left(x, C_{i}\right)} \sinh f\left(x, C_{i}\right) t$

$\gamma(t)=|\vec{r}(t)|-w(x, t)$.

$\tau(x) \equiv \frac{1}{f\left(x, C_{i}\right)} \ln \left\{\begin{array}{c}x-\sqrt{x^{2}-\left|\vec{r}_{0}\right|^{2}+\frac{\left|\vec{v}_{0}\right|^{2}}{\left(f\left(x, C_{i}\right)\right)^{2}}} \\ \left|\vec{r}_{0}\right|+\frac{\left|\vec{v}_{0}\right|}{f\left(x, C_{i}\right)}\end{array}\right\}$

$1 \mathrm{AU}=149 \times 10^{6} \mathrm{~km}$. 


$$
\begin{aligned}
& 1000015551355 \quad t E 21600903
\end{aligned}
$$

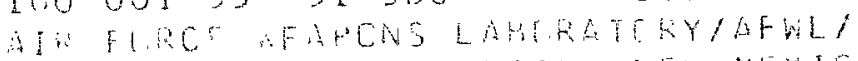

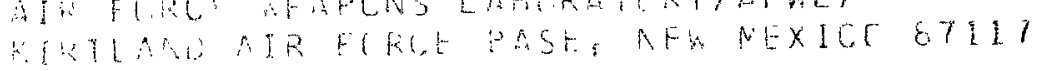

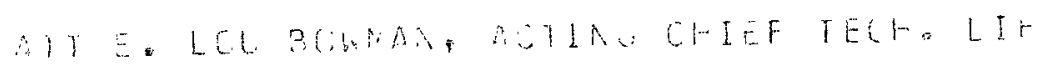

"The aeronautical and space activities of the United States shall be conducted so as to contribute ... to the expansion of buman knowledge of phenomena in the atmosphere and space. The Administration shall provide for the widest practicable and appropriate dissemination of information concerning its activities and the results thereof."

- National Aeronautics and SpaCe ACt of 1958

\section{NASA SCIENTIFIC AND TECHNICAL PUBLICATIONS}

TECHNICAL REPORTS: Scientific and technical information considered important, complete, and a lasting contribution to existing knowledge.

TECHNICAL NOTES: Information less broad in scope but nevertheless of importance as a contribution to existing knowledge.

TECHNICAL MEMORANDUMS:

Information receiving limited distribution because of preliminary data, security classification, or other reasons.

CONTRACTOR REPORTS: Scientific and technical information generated under a NASA contract or grant and considered an important contribution to existing knowledge.
TECHNICAL TRANSLATIONS: Information published in a foreign language considered to merit NASA distribution in English.

SPECIAL PUBLICATIONS: Information derived from or of value to NASA activities. Publications include conference proceedings, monographs, data compilations, handbooks, sourcebooks, and special bibliographies.

\section{TECHNOLOGY UTILIZATION}

PUBLICATIONS: Information on technology used by NASA that may be of particular interest in commercial and other non-aerospace applications. Publications include Tech Briefs, Technology Utilization Reports and Notes, and Technology Surveys.

Details on the availability of these publications may be obtained from:

\section{SCIENTIFIC AND TECHNICAL INFORMATION DIVISION NATIONAL AERONAUTICS AND SPACE ADMINISTRATION Washington, D.C. 20546}

